\title{
INFRARED OBSERVATIONS OF MASS LOSS FROM MASSIVE STARS
}

\author{
M.J. BARLOW \\ Department of Physics \& Astronomy \\ University College London \\ Gower St., London WC1E 6BT.
}

\begin{abstract}
The future use of space-borne IR spectroscopy to determine the ionization structure and abundances in the outer winds of WR stars is described. A mass loss rate of $1.7 \times 10^{-5} \mathrm{M}_{\odot}$ $\mathrm{yr}^{-1}$ has been derived from $10 \mu \mathrm{m}$ photometry of the WO2 star Sanduleak 5 (WR 142). The He/H number ratios in the winds of $\mathbf{P} \mathbf{C y g}$ and $\mathrm{AG}$ Car have been derived from a recombination line analysis of their 1-4 $\mu \mathrm{m}$ spectra and mass loss rates of $2.2 \times 10^{-5} \mathrm{M}_{\odot} \mathrm{yr}^{-1}$ and $3.7 \times 10^{-5} \mathrm{M}_{\odot} \mathrm{yr}^{-1}$ have been respectively derived for them.
\end{abstract}

\section{Introduction}

In this paper I describe a number of recent developments in the field of infrared spectrsocopy and photometry that are relevant to the study of WR and related massive stars. Section 2 describes how spectrophotometry of infrared fine structure lines can be used to derive terminal velocities and ionic abundances in the outer regions of winds. In Section 3, the mass loss rate of a WO star is determined for the first time. In Section 4, the $\mathrm{He} / \mathrm{H}$ ratios and mass loss rates of P Cyg and AG Car are derived.

\section{Wind Terminal Velocities and Abundances from IR Fine Structure Lines}

Aitken, Roche \& Allen (1982) discovered the presence of the IR fine structure lines of [S IV] $10.5 \mu \mathrm{m}$ and [Ne II] $12.8 \mu \mathrm{m}$ in the 8-13 $\mu \mathrm{m}$ spectrum of $\gamma$ Velorum, while van der Hucht \& Olnon (1985) found the [Ne III] $15.5 \mu \mathrm{m}$ line in the IRAS LRS spectrum of the same star. These fine structure lines originate at very large radii in the wind, their emission peaking in the wind regions corresponding to their critical densities of $10^{4}-10^{6} \mathrm{~cm}^{-3}$. At these distances, the stellar wind is moving at terminal velocity and so the line profiles should be flat-topped, with FWZI's equal to twice the wind terminal velocity. Barlow, Roche \& Aitken (1988) confirmed that the [Ne II] $12.8 \mu \mathrm{m}$ line in the spectrum of $\gamma$ Velorum did have a flat-topped profile and determined a wind terminal velocity of $1520 \pm 200 \mathrm{~km} \mathrm{~s}^{-1}$ for the WC8 star responsible for the emission. The terminal velocity of this star had previously been uncertain due to the presence of the $09.5 \mathrm{I}$ companion spectrum in the ultraviolet. Recently, Prinja et al. (1990) have derived a terminal velocity of $1415 \mathrm{~km} \mathrm{~s}^{-1}$ for the wind of the WC 8 component of $\gamma$ Vel, from the black absorption edge velocity of the C IV $1549 \AA$ profile, in good agreement with the IR value. Williams \& Eenens (1989) have also recently shown that the velocity of the displaced absorption component of He I $2.058 \mu \mathrm{m}$ in WR 
spectra yields the wind terminal velocity. Spectroscopy of the $2 \mu \mathrm{m}$ He I line, or of the various IR fine structure lines, can therefore provide a means in the future for determining wind terminal velocities for the numerous WR stars which are too heavily reddened for high resolution UV spectroscopy to be feasible.

The fluxes of the infrared fine structure lines emitted by stars with high mass loss rates can be used, together with the free-free continuum fluxes emitted by the same regions of the wind at radio wavelengths, to derive accurate ionic abundance ratios; relative to hydrogen in the case of OB stars, or relative to helium in the case of WR stars. Barlow et al. (1988) derived $\mathrm{Ne}^{2+} / \mathrm{Ne}^{+}=3.8$ for the outer wind of $\gamma$ Vel, from the fluxes in the [Ne III] $15.5 \mu \mathrm{m}$ and [Ne II] $12.8 \mu \mathrm{m}$ lines, and derived a total neon mass fraction that was a factor of two larger than the solar value. However, evolutionary models for WC stars (e.g. Maeder 1984) predict that neon should be enhanced by a factor of twelve relative to its solar abundance, due to the conversion of all the initial $\mathrm{C}, \mathrm{N}$ and $\mathrm{O}$, first into ${ }^{14} \mathrm{~N}$ by the $\mathrm{CNO}$ cycle during the H-burning phase, and then into ${ }^{22} \mathrm{Ne}$ by successive $\alpha$-particle captures onto ${ }^{14} \mathrm{~N}$ during the He-burning phase. There is therefore a large discrepancy between observations and current theoretical predictions for the neon abundance in WC stars.

A significant advance in our understanding of abundances and ionization structures in the outer winds of massive stars should occur with the advent of ESA's Infrared Space Observatory (ISO), currently scheduled for launch in mid-1993. ISO will carry two spectrometers, one covering the 3-45 $\mu \mathrm{m}$ wavelength region with a resolving power of $1-2 \times 10^{3}$ and the other covering the 45-200 $\mu \mathrm{m}$ wavelength region with resolving powers of 250 or $10^{4}$. A number of fine structure lines of $\mathrm{C}, \mathrm{N}, \mathrm{O}$ and $\mathrm{Ne}$ that are not observable from the ground will therefore become accessible to these spectrometers: [C II] $158 \mu \mathrm{m}$; [N II] $122 \mu \mathrm{m}$ and [N III] $57 \mu \mathrm{m}$; [O I] $63 \& 146 \mu \mathrm{m}$, [O III] $52 \& 88 \mu \mathrm{m}$ and [O IV] $26 \mu \mathrm{m}$; plus [Ne III] $15.5 \& 36 \mu \mathrm{m}$ and [Ne IV] $14.3 \& 24 \mu \mathrm{m}$. With projected ISO sensitivities, it should be possible to derive ionic and elemental abundances from these lines for WN and WC stars out to distances of at least $3 \mathrm{kpc}$ from the Sun. The same should also be true for high luminosity B supergiants - the line fluxes are proportional to $\left(\dot{M} / v_{\infty}\right)^{3 / 2}$ (Barlow et al. 1988), so the lower mass loss rates of these stars, relative to WR stars, are compensated for by their lower terminal velocities.

\section{The Mass Loss Rate of the WO2 star WR 142}

The WO stars are a rare group of WR stars whose optical spectra show very strong O VI 3811,34 $\AA$ emission. Two WO stars have been identified within our own Galaxy but to date no radio detections have been obtained that would allow their mass loss rates to be derived. Given that these objects appear to be the most evolved WR stars so far identified, their mass loss rates are clearly of interest. It is now possible to estimate the mass loss rate for the closest WO star, Sanduleak 5 (三WR $142 \equiv$ ST 3), using infrared photometry obtained with the $3.8 \mathrm{~m}$ UKIRT.

The mean infrared-radio spectral index $(10 \mu \mathrm{m}-6 \mathrm{~cm})$ found for WR stars is about 0.75 (Bieging et al. 1982), compared to the value of 0.60 predicted for an optically thick stellar wind of constant degree of ionization (Wright \& Barlow 1975). It has been realised recently (e.g. Schmutz \& Hamann 1986; Hillier 1987) that the main reason for this discrepancy is that the degree of ionization in WR winds decreases outwards, so that the effective charge per emitting (helium) ion is 2 in the IR-emitting region, but only 1 in the radio-emitting region. It is therefore valid to derive WR mass loss rates from $10 \mu \mathrm{m}$ free-free excess fluxes, 


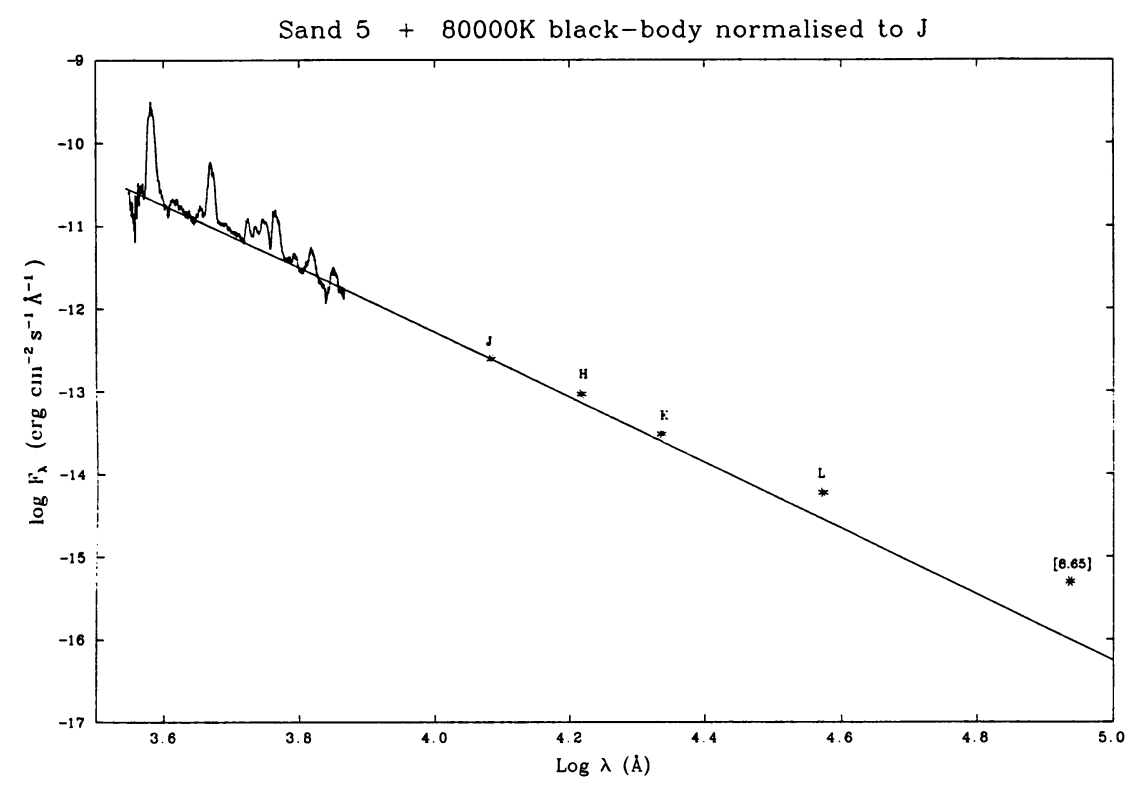

Figure I

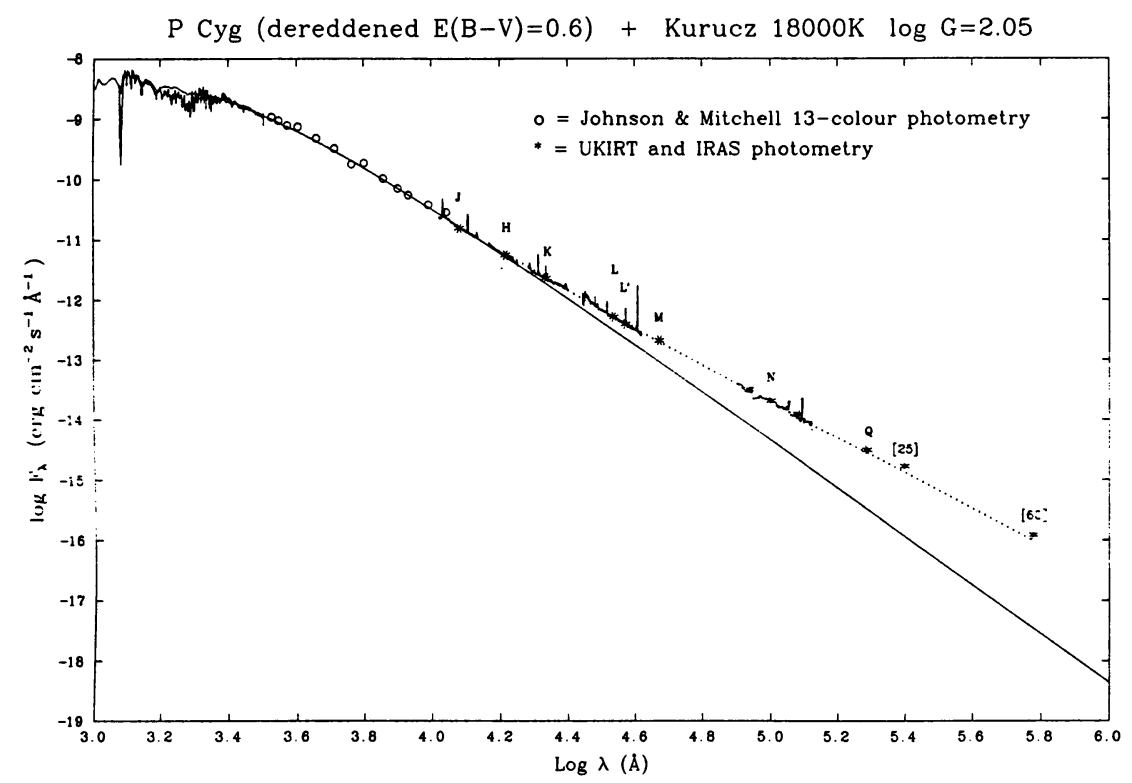

Figure 2 
provided the appropriate wind composition and degree of ionization are adopted.

Sanduleak 5 is located in the heavily obscured Galactic cluster Be 87, at a distance of 1 kpc (Turner \& Forbes 1982). Figure 1 shows its optical-IR energy distribution after dereddening by $A_{V}=6.32$ magnitudes. The optical flux distribution comes from Barlow \& Hummer (1982), the JHKL' data comes from Williams (1982) and the $8.65 \mu \mathrm{m}$ datum comes from UKIRT. After subtraction of an $80,000 \mathrm{~K}$ blackbody normalised to the optical and $\mathrm{J}$ data (Figure 1), an excess flux of $0.10 \mathrm{Jy}$ at $8.65 \mu \mathrm{m}$ is derived. For a radio-IR spectral index of 0.75 , a $6 \mathrm{~cm}$ radio free-free flux of $0.13 \mathrm{mJy}$ is predicted from Sanduleak 5 , compared to the upper limit of $0.6 \mathrm{mJy}$ set by Abbott et al. (1986). To estimate its mass loss rate, a wind terminal velocity of $5500 \mathrm{~km} \mathrm{~s}^{-1}$ is adopted, along with $\mathrm{n}\left(\mathrm{C}^{4+}\right) / \mathrm{n}\left(\mathrm{He}^{2+}\right)=$ 0.20 and $\mathrm{n}\left(\mathrm{O}^{6+}\right) / \mathrm{n}\left(\mathrm{He}^{2+}\right)=0.03$ (Kingsburgh \& Barlow 1990). A temperature of $40,000 \mathrm{~K}$ is adopted for the IR-emitting region of the wind (the Gaunt factor is very weakly dependent upon this parameter). A mass loss rate of $1.68 \times 10^{-5} \mathrm{M}_{\odot} \mathrm{yr}^{-1}$ is derived.

This mass loss rate is at the lower end of the range found for WR stars. The average found for those WC stars having well-determined radio fluxes and terminal velocities (Prinja et al. 1990 ) is $6 \times 10^{-5} \mathrm{M}_{\odot} \mathrm{yr}^{-1}$. However, the mass loss rate found by the latter authors for the WC5 star HD $165763,3.2 \times 10^{-5} \mathrm{M}_{\odot} \mathrm{yr}^{-1}$, is intermediate between the WC mean mass loss rate and the rate found above for Sanduleak 5. Since HD 165763 has the earliest spectral type (and the one closest to that of Sand. 5) amongst the WC stars with a radio-derived mass loss rate, one might speculate that this is consistent with WR stars having a mass loss rate which is a strong function of mass (Abbott et al. 1986; Langer 1989), since the mass loss rate of a WR star should then decline as it evolves by mass loss stripping from a WC to WO type.

\section{The He/H Ratios and Mass Loss Rates of P Cyg and AG Car}

The mean $6 \mathrm{~cm}$ radio flux for P Cygni is $6.7 \pm 0.2 \mathrm{mJy}$ (van den Oord al. 1985), its wind electron temperature in the radio-emitting region is $6000 \mathrm{~K}$ (Becker \& White 1985) and its wind terminal velocity is $206 \mathrm{~km} \mathrm{~s}^{-1}$ (Lamers et al. 1985). For a distance of $1.8 \mathrm{kpc}$, its mass loss rate is therefore $7.2 \times 10^{-6} \mu \mathrm{M}_{\odot} \mathrm{yr}^{-1}$, where $\mu$ is the mean mass per ion in the radio emitting zone. The emitting ions are assumed to be singly ionized. However, Drew (1985) has shown that helium will be neutral beyond about 5 stellar radii in the wind of $\mathrm{P}$ Cyg, i.e. in the radio-emitting region, so $7.2 \times 10^{-6} \mathrm{M}_{\odot} \mathrm{yr}^{-1}$ is only the hydrogen mass loss rate of $\mathrm{P} \mathrm{Cyg}$, and to determine its total mass loss rate we need to know the $\mathrm{He} / \mathrm{H}$ abundance ratio, and thus $\mu$, in its wind.

Figure 2 shows the UV-IR energy distribution of P Cyg, from Deacon \& Barlow (1990). Shown are the flux-calibrated IUE high-resolution data, Johnson \& Mitchell 13-colour optical photometry, UKIRT and IRAS $1.25-60 \mu \mathrm{m}$ photometry and 1-4 $\mu \mathrm{m}$ spectrophotometry. The flux distribution has been dereddened by $\mathrm{E}(\mathrm{B}-\mathrm{V})=0.60$, and the UV-1.25 $\mu \mathrm{m}$ flux distribution has been fitted by an $18000 \mathrm{~K}, \log g=2.05$ LTE model, enabling the exccess $\mathrm{f}-\mathrm{f}$ and $b-f$ fluxes due to the wind to be derived for wavelengths longwards of $2 \mu \mathrm{m}$. The excess flux at $10 \mu \mathrm{m}$ is $5.4 \mathrm{Jy}$. A stellar radius of $92.5 \mathrm{R}_{\odot}$ is implied by the model atmosphere normalisation, corresponding to a luminosity of $8.1 \times 10^{5} \mathrm{~L}_{\odot}$.

Cohen \& Barlow (1980) published IR photometry out to $10 \mu \mathrm{m}$ for AG Car, obtained on March 31 1977. Hutsemekers \& Kohoutek (1988) have published a long-term light curve for AG Car, along with high-resolution optical spectroscopy acquired on February 22/23 1977, only one month before the IR photometry of Cohen \& Barlow; this showed AG Car at the 
time to have had a spectrum very similar to that of $\mathrm{P}$ Cyg. In addition, the flat-topped [Fe II] lines in the optical spectrum of AG Car yielded a wind expansion velocity of 205 $\mathrm{km} \mathrm{s}^{-1}$, almost identical to that of $\mathrm{P}$ Cyg. After dereddening by $\mathrm{E}(\mathrm{B}-\mathrm{V})=\mathbf{0 . 6 3}$ (Humphreys et al. 1989) and normalising an LTE $17000 \mathrm{~K}, \log \mathrm{g}=1.9$ model to the $\mathrm{J}$ photometry, an excess flux of $1.35 \mathrm{Jy}$ at $10 \mu \mathrm{m}$ is derived (Deacon \& Barlow 1990). For a distance to AG Car of $6 \mathrm{kpc}$ (Humphreys et al. 1989), the model atmosphere normalisation implies a stellar radius of $121.8 \mathrm{R}_{\odot}$ and a luminosity of $1.12 \times 10^{6} \mathrm{~L}_{\odot}$.

Since the spectrum and wind terminal velocity of AG Car at the epoch of the IR photometry were so similar to those of $\mathbf{P}$ Cyg, it seems reasonable to assume similar wind velocity laws, in which case the ratio of their mass loss rates is determined by the ratio of their absolute excess fluxes at $10 \mu \mathrm{m}$. The $10 \mu \mathrm{m}$ excess flux for P Cyg is 4.0 times that for AG Car, while the square of the distance to AG Car is 11.1 times larger than that to P Cyg. Therefore, from Wright \& Barlow (1975), $(\dot{M} / \mu)^{4 / 3}$ for AG Car is 2.78 times larger than that for $\mathrm{P}$ Cyg. To compare their mass loss rates, we need to know the $\mathrm{He} / \mathrm{H}$ ratios, and thus the values of $\mu$, in each of their winds.

The $\mathrm{He}^{+} / \mathrm{H}^{+}$ratio in the IR-emitting region of the wind of $\mathrm{P}$ Cygni can be derived from an analysis of the He I and $H$ I recombination line fluxes observed in the 1-4 $\mu \mathrm{m}$ spectra shown in Figure 2. These spectra, obtained as service observations with CGS 2 at UKIRT, have a resolving power of 300-600, depending on the wavelength region. The recombination coefficients of Hummer \& Storey (1987 for H I; in preparation for He I) were used, with $\mathrm{T}_{e}=15000 \mathrm{~K}$ and $\mathrm{n}_{e}=10^{11} \mathrm{~cm}^{-3}$. In order to avoid optical depth self-absorption effects, only $\mathrm{H}$ I recombination lines with $\Delta \mathrm{n}>3$ were used - this left eight $\mathrm{H}$ I IR lines suitable for the analysis. Four He I lines between 1.2-2.5 $\mu \mathrm{m}$ were used - they corresponded to angular momentum sub-components of the 4-3,5-3 and 6-4 terms. The 2s-2p lines of He I at $1.083 \mu \mathrm{m}$ and $2.058 \mu \mathrm{m}$ were not used, as these transitions are strongly affected by optical depth and collisional excitation effects. A $\mathrm{He}^{+} / \mathrm{H}^{+}$ratio of $0.5 \pm 0.1$, by number, was derived for the IR emitting zone of P Cyg. A value of $\mu(\mathrm{IR})=2.0$ results, while $\mu$ (radio) $=3.0$, since helium is neutral there. The total mass loss rate for $\mathrm{P}$ Cyg given by the radio data and $\mu=3.0$ is therefore $2.16 \times 10^{-5} \mathrm{M}_{\odot} \mathrm{yr}^{-1}$.

McGregor et al. (1988) obtained JHK spectroscopy of AG Car with a resolving power of 500 during 1984 and 1985 . Their March 1984 spectra were taken at an epoch when AG Car had a similar V magnitude to 1977 February/March, according to the light curve of Hutsemekers \& Kohoutek (1988). The line fluxes measured by McGregor et al. from their March $1984 \mathrm{JHK}$ spectra have therefore been analysed in the same manner as described above for P Cyg. Three H I and two He I lines were suitable for the analysis and yielded $\mathrm{He}^{+} / \mathrm{H}^{+}=0.25 \pm 0.08$, by number, for the IR-emitting region of AG Car. This corresponds to $\mu(\mathrm{IR})=1.6$.

$\dot{M} / \mu$ was found above to be 2.15 times larger for AG Car than for P Cyg. Since $\mu($ IR) is 1.25 times larger for P Cyg than for AG Car, the mass loss rate of AG Car in 1977 March must therefore have been 1.7 times larger than that of $\mathrm{P}$ Cyg, implying $\dot{M}=3.7 \times 10^{-5} \mathrm{M} \odot$ $\mathrm{yr}^{-1}$ for AG Car.

Maeder \& Meynet (1987) and Langer (1990) have found that a star of $60 \mathrm{M}_{\odot}$ initial mass should evolve into a WNL-type star when the surface hydrogen mass fraction, $\mathrm{X}_{H}$, drops below $0.23-0.30$. The analysis above implies that $\mathrm{X}_{H}=0.5$ for AG Car, while $\mathrm{X}_{H}=0.33$ for P Cyg. Therefore both stars are well on their way to becoming WNL stars according to this interpretation, consistent with the proposals by Lamers et al. (1983) and Humphreys et al. (1989) that P Cyg and AG Car are currently evolving towards the WN stage. 


\section{References}

Abbott, D. C., Bieging, J. H., Churchwell, E. \& Torres, A. V., 1986. Astrophys. J., 303, 239.

Aitken, D. K., Roche, P. F. \& Allen, D. A., 1982. Mon. Not. R. astr. Soc., 200, 69P.

Barlow, M. J. \& Hummer, D. G., 1982. IAU Symp. No. 99, p. 387, eds. de Loore, C. W. H. \& Willis, A. J., Reidel, Dordrecht, Holland.

Barlow, M. J., Roche, P. F. \& Aitken, D. K., 1988. Mon. Not. R. astr. Soc., 232, 821.

Becker, R. H. \& White, R. L., 1985. Radio Stars, p. 139, eds. Hjellming, R. M. \& Gibson, D. M., Reidel, Dordrecht, Holland.

Bieging, J. H., Abbott, D. C. \& Churchwell, E. B., 1982. Astrophys. J., 263, 207.

Cohen, M. \& Barlow, M. J., 1980. Astrophys. J., 238, 585.

Deacon, J. R. \& Barlow, M. J., 1990. These Proceedings.

Drew, J. E., 1985. Mon. Not. R. astr. Soc., 217, 867.

Hillier, D. J., 1987. Astrophys. J. Suppl., 63, 947.

van der Hucht, K. A. \& Olnon, F. M., 1985. Astr. Astrophys., 149, L17.

Hummer, D. G., Barlow, M. J. \& Storey, P. J., 1982. IAU Symp. No. 99, p. 79, eds. de Loore, C. W. H. \& Willis, A. J., Reidel, Dordrecht, Holland.

Hummer, D. G. \& Storey, P. J., 1987. Mon. Not. R. astr. Soc., 224, 801.

Humphreys, R. M., Lamers, H. J. G. L. M., Hoekzema, N. \& Cassatella, A., 1989. Astr. Astrophys., 218, L17.

Hutsemekers, D. \& Kohoutek, L., 1988. Astr. Astrophys., 73, 217.

Kingsburgh, R. L. \& Barlow, M. J., 1990. These Proceedings.

Lamers, H. J. G. L. M., de Groot, M. J. H. \& Cassatella, A., 1985. Astr. Astrophys., 128, L8.

Lamers, H. J. G. L. M., Korevaar, P. \& Cassatella, A., 1985. Astr. Astrophys., 149, 29.

Langer, N., 1989. Astr. Astrophys., 220, 135.

Langer, N., 1990. Properties of Hot Luminous Stars, p. 328, ed. Garmany, C. D., Astr. Soc. Pacific Conf. Series, Vol. 7.

Maeder, A., 1984. Astr. Astrophys., $120,113$.

Maeder, A. \& Meynet, G., 1987. Astr. Astrophys., 182, 243.

McGregor, P. J., Hyland, A. R. \& Hillier, D. J., 1988. Astrophys. J., 324, 1071.

van den Oord, G. H. J., Waters, L. B. F. M., Lamers, H. J. G. L. M., Abbott, D. C.,

Bieging, J. H. \& Churchwell, E., 1985. Radio Stars, eds. Hjellming, R. M. \& Gibson,

D. M., Reidel, Dordrecht, Holland.

Prinja, R. K., Barlow, M. J. \& Howarth, I. D., 1990. Astrophys. J., 361.

Schmutz, W. \& Hamann, W.-R., 1986. Astr. Astrophys., 166, L11.

Turner, D. G. \& Forbes, D., 1982. Publ. astr. Soc. Pacific, 94, 789.

Williams, P. M., 1982. Mon. Not. R. astr. Soc., 199, 93.

Williams, P. M. \& Eenens, P. R. J., 1989. Mon. Not. R. astr. Soc., 240, 445.

Wright, A. E. \& Barlow, M. J., 1975. Mon. Not. R. astr. Soc., 170, 41. 


\section{DISCUSSION}

Moffat: Another mass estimate of a WO star comes from a study of the orbit of AB8 in the SMC for which the WO4 star has a mass of $\simeq 5 M_{\odot}$. With Sand 5 this confirms that WO stars do have relatively low masses, regardless of the ambient metallicity.

Barlow: Yes. In addition, the poster paper by Kingsburgh and Barlow shows that the 0 star component of AB8 (= Sk $188=$ Sand 1) has a spectral type of $07 \pm 0.5$. If it is a dwarf, then the implied mass for the WO4 component would be consistent with your new estimate.

Taylor: We have monitored P Cyg (spectropolarimetrically) during its recent outburst. These data show that there is a non-steady mass outflow and the wind is non-spherically symmetric. We expect that there is a circumstellar wind with intermittent "blob" ejections. Barlow: The high resolution IUE observations of Lamers et al. (1985) showed moving "shell" in the wind of P Cyg, which might be related to the non-steady behaviour that you observed. The high spatial resolution VLA data of Becker and White (1985) indicate that, out in the radio emitting zone, the wind of P Cyg is fairly symmetric. Schmidt-Burgk (1982) has shown that very large asymmetry factors are needed before radio-derived mass loss rates are seriously affected.

Leitherer: AG Car is known to be surrounded by a jet-like structure indicating strong departures from spherical symmetry (binary, magnetic field?). P Cyg in contrast is believed to have a spherically symmetrical wind. This comes from VLA observations of the wind. Did you take the different geometry into account for the $\dot{M}$ you derive?

Barlow: The assumption made was that AG Car had the same distribution of matter in its wind as P Cyg. The Australia Telescope should shortly carry out similar observations on AG Car as the VLA did on P Cyg. AG Car is certainly near to a jet-like structure, but it is also of course surrounded by a remarkably symmmetric ring nebula. 


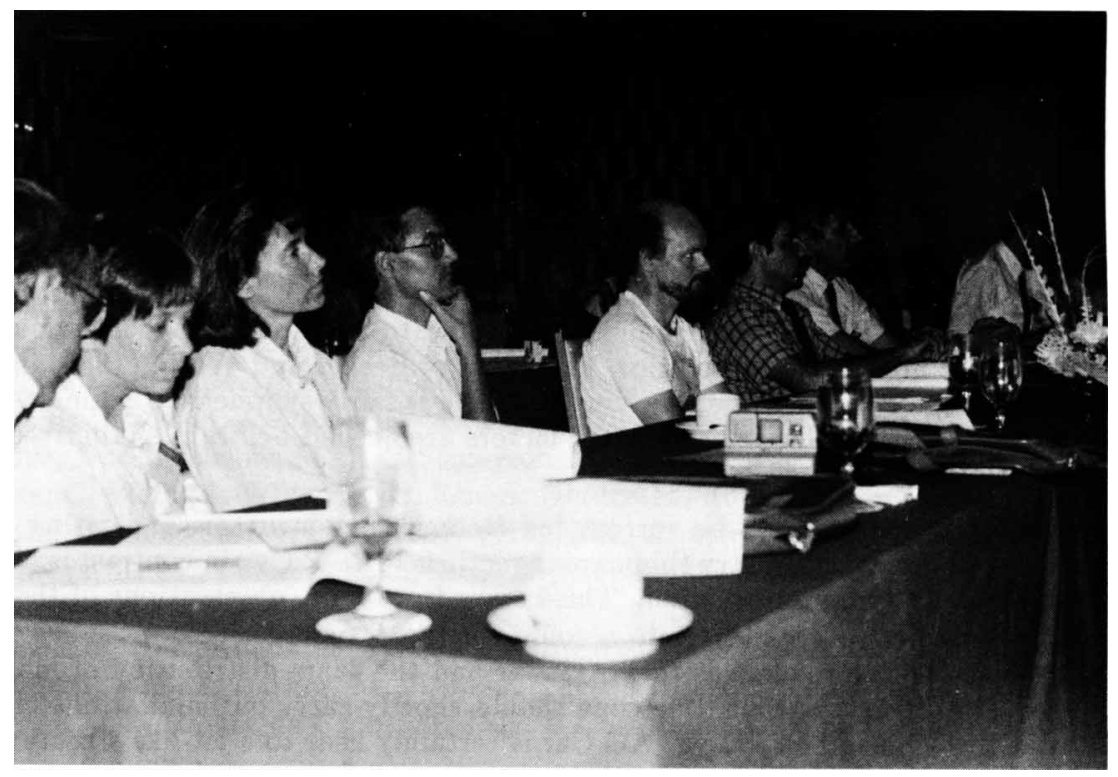

Cassinelli, Taylor, Schulte-Ladbeck, Leitherer, Barlow, Cohen, Felli, Walborn 